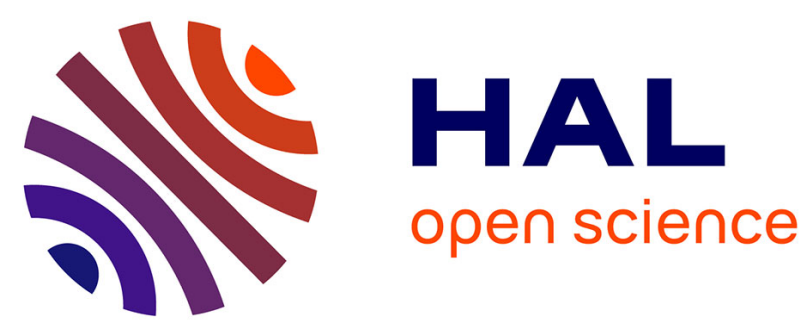

\title{
Irisin levels in LMNA-associated partial lipodystrophies
} F. Bensmaïne, K. Benomar, S. Espiard, C. Vahe, K. Le Mapihan, G. Lion, M. Lemdani, E. Chazard, O. Ernst, C. Vigouroux, et al.

\section{To cite this version:}

F. Bensmaïne, K. Benomar, S. Espiard, C. Vahe, K. Le Mapihan, et al.. Irisin levels in LMNA-associated partial lipodystrophies. Diabetes \& Metabolism, 2019, 45, pp.67 - 75. 10.1016/j.diabet.2018.08.003 . hal-03485631

\section{HAL Id: hal-03485631 \\ https://hal.science/hal-03485631}

Submitted on 20 Dec 2021

HAL is a multi-disciplinary open access archive for the deposit and dissemination of scientific research documents, whether they are published or not. The documents may come from teaching and research institutions in France or abroad, or from public or private research centers.
L'archive ouverte pluridisciplinaire HAL, est destinée au dépôt et à la diffusion de documents scientifiques de niveau recherche, publiés ou non, émanant des établissements d'enseignement et de recherche français ou étrangers, des laboratoires publics ou privés.

\section{다)(1) $(5$}

Distributed under a Creative Commons Attribution - NonCommerciall 4.0 International 


\title{
IRISIN LEVELS IN LMNA-ASSOCIATED PARTIAL LIPODYSTROPHIES
}

\author{
Faiza BENSMAÏNEE ${ }^{1}$, Kanza BENOMAR $^{1}$, Stéphanie ESPIARD ${ }^{1}$, Claire VAHE ${ }^{1}$, Kristell LE \\ MAPIHAN ${ }^{1}$, Georges LION $^{2}$, Mohamed LEMDANI ${ }^{3}$, Emmanuel CHAZARD $^{3}$, Olivier ERNST ${ }^{4}$, \\ Corinne VIGOUROUX ${ }^{5,6}$, Pascal PIGNY ${ }^{7 *}$, Marie-Christine VANTYGHEM ${ }^{1,6,8 *}$
}

* These two authors have contributed equally.

\footnotetext{
${ }^{1}$ Department of Endocrinology and Metabolism, Lille University Hospital, 59037 Lille, France

${ }^{2}$ Department of Nuclear Medicine, Lille University Hospital, 59 037, Lille, France

${ }^{3}$ Department of Public Health, Univ. Lille EA 2694, F-59000 Lille, France

${ }^{4}$ Department of Radiology, Lille University Hospital, 59037 Lille France

${ }^{5}$ Assistance Publique-Hôpitaux de Paris (AP-HP), Saint-Antoine Hospital, Departments of Endocrinology and Molecular Biology and Genetics, Sorbonne Université, INSERM UMR_S938, Saint-Antoine Research Centre, F-75012 Paris, France, and ICAN (Institute of Cardiometabolism and Nutrition), F-75013 Paris, France

${ }^{6}$ National Reference Centre of Rare Diseases of Insulin Secretion and of Insulin Sensitivity, hospital Saint Antoine, Paris

${ }^{7}$ Department of Biology, Lille University Hospital, 59 037, Lille France

${ }^{8}$ Inserm U1190, European Genomic Institute for Diabetes, F-59000 Lille, France
}

\section{Corresponding author and reprint requests:}

Prof. M.-C. Vantyghem, MD, PhD

Endocrinology and Metabolism Department, 1, rue Polonovski, C Huriez Hospital, Lille University Hospital, 59037 Lille Cedex, France

Tel: +33 3204445 35; Fax: +33 320446985

E-mail: mc-vantyghem@chru-lille.fr

Received 1 May 2017; Accepted 16 August 2018

Abbreviations: BMI: body mass index; DXA: dual-energy X-ray absorptiometry; FBG: fasting blood glucose; FPLD2: LMNA-related familial partial lipodystrophy; HbA1c: haemoglobin A1c; HC: healthy controls; OND: obese non-diabetic 


\section{ABSTRACT}

Aim. - The adipo-myokine irisin regulates energy expenditure and fat metabolism. LMNA-associated familial partial lipodystrophy (FPLD2) comprises insulin resistance, muscle hypertrophy and lipoatrophy. The aim of this study was to investigate whether irisin could be a biomarker of FPLD2.

Patients and methods. - This case-control study included 19 FPLD2 subjects, 13 obese non-diabetic (OND) patients and 19 healthy controls (HC) of normal weight (median BMI: 26, 39 and $22 \mathrm{~kg} / \mathrm{m}^{2}$, respectively). Serum irisin and leptin levels, body composition (DXA/MRI) and metabolic/inflammatory parameters were compared in these three groups.

Results. - BMI and MRI intra-abdominal fat significantly differed among these three groups, whereas DXA total fat mass and leptin levels were higher in the OND group, but did not differ between HC and FPLD2. Lipodystrophy patients had higher intra-abdominal/total abdominal fat ratios than the other two groups. Irisin levels were higher in FPLD2 and OND patients than in HC (medians: 944, 934 and $804 \mathrm{ng} / \mathrm{mL}$, respectively). However, irisin/leptin ratios and lean body mass percentages were strikingly higher, and lean mass indices lower, in FPLD2 and HC than in the OND (median irisin/leptin ratios: 137, 166 and 21, respectively). In the entire study group, irisin levels positively correlated with BMI, lean body mass and index, intra-abdominal/total abdominal fat ratio, triglyceride, cholesterol, insulin, glucose and HbA1c levels. Also, intra-abdominal/total abdominal fat ratio and lean body mass better differentiated the three groups only in female patients.

Conclusion. - Circulating irisin is similarly increased in FPLD2 and OND patients, who are characterized by higher lean body mass regardless of their clearly different fat mass. However, irisin/leptin ratios, strikingly higher in FPLD2 than in OND patients, could help to make the diagnosis and prompt genetic testing in clinically atypical cases.

Key words: Fat mass; Irisin; Lamin A; Lean mass; Leptin; Lipodystrophy; Muscle; Obesity 


\section{INTRODUCTION}

The spectrum of adipose tissue diseases ranges from obesity to lipodystrophy, and is usually defined according to the amount and distribution of fat mass. Obesity features an increased amount of fat and is characterized by a body mass index $(\mathrm{BMI})>30 \mathrm{~kg} / \mathrm{m}^{2}$. In contrast, lipodystrophy syndromes are characterized not only by a decrease and abnormal distribution of total fat mass, but also, in most cases, by a very muscular appearance associated with severe insulin resistance, diabetes and hypertriglyceridaemia [1-3]. This group of rare diseases may be acquired or inherited, generalized or partial. Among them, LMNA-related familial partial lipodystrophy (FPLD2) is characterized by progressive fat loss in the extremities at puberty, with muscular hypertrophy predominantly in the calves [4-6]. Lamin A, encoded by the LMNA gene, is a member of the intermediate-filament protein family and required for nuclear lamina formation. The hotspot mutation at codon $R 482$ could affect the ability of A-type lamins to bind DNA and transcription factors and, subsequently, alter adipocyte differentiation and skeletal muscle metabolism [7-10]. Fat remodelling, including altered partitioning of white (energy-storing) and brown/beige (thermogenic) fat, has been described in lipodystrophy patients with $L M N A$ mutations $[10,11]$. Interestingly, brown adipocytes are thought to originate from a cell precursor shared with skeletal muscle, whereas white adipocytes originate from distinct precursors $[12,13]$.

The diagnosis of lipodystrophy is clinically based, but may be difficult, while FPLD2 may have a greater prevalence than is currently believed due to lack of recognition of its more subtle forms [14]. Indeed, screening all non-obese patients with type 2 diabetes mellitus (T2DM) for known FPLD genes increases the prevalence of lipodystrophy by more than 400 -fold from their baseline assessment [15-17]. However, there are as yet no validated biological markers to date for identifying FPLD, although a decrease of leptin levels correlates with a decrease of fat mass in lipodystrophy [18].

Irisin is a newly discovered adipo-myokine involved in myogenesis and in the 'browning' of white adipose tissue $[19,20]$. A cleaved and secreted fragment of fibronectin type III domaincontaining 5 (FNDC5), irisin stimulates muscle growth and browning of white adipocytes in both mice and humans [21-23]. In humans, circulating irisin levels, correlated with lean body mass, increase after exercise in healthy young individuals, and are also associated with markers of adiposity, 
components of the metabolic syndrome, cardiovascular events and polycystic ovary disease [24-30]. Yet, levels of serum irisin have not been investigated in human lipodystrophy syndromes associating muscle hypertrophy and adipocyte dysfunction.

Thus, the objective of the present study was to identify simple biomarkers of FPLD2. Our hypothesis was that an imbalance between lean and fat mass in FPLD2 might trigger specific deregulation of adipokines and myokines. To this end, it was necessary to determine: (i) lean and fat body masses in FPLD2 subjects, in obese non-diabetic (OND) patients and in normal-weight healthy controls (HC); (ii) serum levels of irisin and leptin in FPLD2 vs OND patients and HC; and (iii) the usefulness of these markers for FPLD2 diagnosis.

\section{PATIENTS AND METHODS}

\section{Study design}

In this case-control study, control subjects and patients were recruited from a clinical trial analyzing the clinical, metabolic and cytokine profiles of adult patients who had either a qualitative (lipodystrophy syndrome) or quantitative (obesity) anomaly of adipose tissue (Clin.gov2009-AO1169-48/PHRC 2009_09/094). The study protocol was approved by the relevant ethics committee, and all selected subjects gave their written informed consent to participate. Thus, the present study included men and women aged $>18$ years from the following groups: (i) patients with FPLD2 due to LMNA mutations (FPLD2); (ii) OND patients (BMI > $30 \mathrm{~kg} / \mathrm{m}^{2}$ ) according to American Diabetes Association criteria after an oral glucose tolerance test; and (iii) normal-weight $\mathrm{HC}$ (BMI $>18 \mathrm{~kg} / \mathrm{m}^{2}$ but $<25 \mathrm{~kg} / \mathrm{m}^{2}$ ). Patients were excluded if they were aged $<18$ years or fulfilled any of the following criteria: $\mathrm{BMI}>60 \mathrm{~kg} / \mathrm{m}^{2}$; creatinine $>1.5 \mathrm{mg} / \mathrm{dL}$; autoimmune disorders; active cancer; excessive alcohol consumption; coagulation disorders; active chronic infection, including human immunodeficiency virus (HIV) and hepatitis C; treatment that might interfere with metabolic function, including oestrogens and analogues (contraceptive pill, tamoxifen); and other medicolegal conditions (psychiatric disease; pregnant or breastfeeding women; patients unable to provide informed consent or refusing to sign consent forms; participation in other studies, thereby precluding their participation in an additional protocol; patients under guardianship or deprived of their freedom rights; patients not 
covered by health insurance). Levels of physical activity in all these subjects was low or moderate ( $\leq$ $30 \mathrm{~min} /$ day).

\section{Patients}

A total of 51 patients (15 males and 36 females), including 19 lipodystrophy patients with LMNA pathogenic variants, 13 OND patients and 19 normal-weight $\mathrm{HC}$ were enrolled from the abovementioned cohort.

\section{Outcomes}

Anthropometric and biological markers were prospectively assessed for each participant at baseline.

\section{Anthropometric and body composition parameters}

Age, gender, height and body weight were recorded, and BMI was calculated. Total body fat and lean masses were measured by dual-energy X-ray absorptiometry (DXA; Lunar DPX-IQ, GE Healthcare, Waukesha, WI, USA). Total and intra-abdominal adipose tissue surface areas to allow assessment of subcutaneous and visceral fat were calculated at the same radiological centre, using 1$\mathrm{cm}$ reconstructed slices of abdominal L4 magnetic resonance imaging (MRI) scans and Extended Brilliance Workspace (EBW) version 3.5 software (Philips Medical Systems, Eindhoven, The Netherlands). Fat and lean body mass measurements in grammes and percentages were used to derive ratios of total fat $/$ height $^{2}$ (body fat mass index) and lean mass $/$ height ${ }^{2}$ (body lean mass index), total and intra-abdominal fat surface areas, and intra-abdominal/total abdominal fat surface areas.

\section{Laboratory parameters}

Fasting blood glucose (FBG), haemoglobin A1c (HbA1c), liver enzymes [aspartate aminotransferase (AST), alanine aminotransferase (ALT), triglycerides, and total, low-density lipoprotein (LDL) and high-density lipoprotein (HDL) cholesterol levels were measured using routine methods. Fasting insulin levels were measured in duplicate at the same centralized laboratory by 
monoclonal immunoradiometric assay (Bi-INS-IRMA; Cisbio, Bedford, MA, USA). Leptin levels were measured by radioimmunoassay using commercial kits (Human Leptin RIA, EMD Millipore, Billerica, MA, USA). Intra- and interassay coefficients of variation $(\mathrm{CVs})$ were $<8.5 \%$. Irisin was assessed from serum stored at $-80^{\circ} \mathrm{C}$ until analysis, using commercially available enzyme-linked immunosorbent assay (ELISA) kits (EK-067-29, Irisin Recombinant, Phoenix Pharmaceuticals Inc., Burlingame, CA, USA) and run in duplicate; intra- and interassay CVs were $<10 \%$ and $<15 \%$, respectively, according to manufacturer's information.

\section{Statistical analysis}

Quantitative variables were expressed as medians, first and third quartiles or means \pm standard deviation (SD). Between-group comparisons were performed using Kruskal-Wallis or Mann-Whitney tests. Qualitative values were expressed as proportions, and compared between groups using chisquared or Fisher's exact tests, according to validity conditions. Confidence intervals (CIs) for proportions were computed using a binomial law. Correlations were assessed by Spearman's rank correlation coefficient and related tests. All tests were two-sided, and a $P$ value $<0.05$ was considered statistically significant. All statistical analyses were performed with the ROCR software package (R Foundation for Statistical Computing, Vienna, Austria).

Finally, an arbitrarily developed score was used to distinguish FPLD2 patients from other patients (OND vs HC), defined by the following equation: FPLD2 score $=$ irisin $-[12.5 \times$ leptin $]$. The sensitivity and specificity of this score were calculated for different thresholds, with genetic diagnosis considered the gold standard. The receiver operating characteristic (ROC) curve was plotted, and the area under the curve (AUC) thus computed: a value of 1 denoted perfect discrimination, whereas a value of 0.5 denoted a random (and therefore useless) prediction of patient status.

\section{RESULTS}

\section{Description of the lipodystrophy group}

The FPLD2 group included patients with LMNA heterozygous pathogenic variants: 14 patients had the well-known c.1444C>T (p.Arg482Trp) or c.1445G>A (p.Arg482Gln) substitutions (12 were women, 
aged 19-75 years, with typical FPLD2 phenotypes); and five patients (including four women) had other LMNA molecular defects [two c.1173dup (p.Ser392Glnfs*34), one c.860del (p.Ala287Valfs*193), one c.1238del (p.Gly413Alafs*67) and one c.1157G>C (p.Arg386Thr) variant), and were aged 26-63 years with less-typical partial lipodystrophy phenotypes. The p.Arg386Thr LMNA variant had previously been reported associated with a mixed phenotype of cardiomyopathy and lipodystrophy [31]. The three remaining $L M N A$ variants had never been previously reported but, as they are thought to drastically modify protein structure, they are also very likely to be diseasecausing.

Ten of the 14 patients with R482 substitutions were treated with antidiabetic drugs, including insulin in four cases. Seven of the 10 R482 patients not treated with insulin had serum insulin levels $>4$ $\mathrm{mIU} / \mathrm{L}$ (5.1-56.6 mIU/L), with FBG levels $>5.5 \mathrm{mmol} / \mathrm{L}$ in 10/14 cases. Two of the five patients bearing other $L M N A$ variants were treated with antidiabetic drugs, but none with insulin. Two of the five non-R482-mutated patients had fasting insulin levels > $4 \mathrm{mIU} / \mathrm{L}(5.1-6.4 \mathrm{mIU} / \mathrm{L})$, with only one patient showing FBG $>5.5 \mathrm{mmol} / \mathrm{L}$.

\section{Comparison of the three groups}

The clinical, biochemical and anthropometric characteristics of our three study groups (FPLD2, OND, HC) are shown in Table I. Age did not differ significantly, but gender differed slightly among the three groups, with lipodystrophy being more easily identified in female than in male patients.

\section{Anthropometric parameters}

Although BMI, proportion of total body fat, body fat index and total abdominal fat area were, as expected, higher in OND vs HC and FPLD2 patients, these parameters did not significantly differ between the HC and FPLD2 except for BMI (Table I). Compared with OND, lean body mass percentages and lean mass index/total abdominal fat surface area ratios were higher in HC and FPLD2 patients $(P<0.0001$ for both). However, the lean mass index was, or tended to be, lower in HC and FPLD2 than in OND patients $(P<0.0001$ and $P=0.08$, respectively), with a value between those in 
the HC and OND $(P=0.12)$ for FPLD2 (Table I, Fig. 1 E). The intra-abdominal/total abdominal fat ratio did not differ significantly between OND and HC groups, but was higher in FPLD2 patients, reflecting their specific subcutaneous lipoatrophy (Table I, Fig. 1 D). In fact, BMI and intra-abdominal fat mass were the best parameters to discriminate among the three groups (Table I, Fig. 1).

\section{Metabolic and inflammatory parameters}

Compared with the HC, levels of HbA1c, insulin, triglycerides, ALT and C-reactive protein (CRP) were higher, or tended to be significantly higher, in OND and FPLD2 patients (Table I). In addition, HbA1c, insulin, triglyceride and ALT levels were even higher in the FPLD2 than in the OND group (Table I).

\section{Irisin and leptin levels}

Leptin levels did not differ significantly between HC and FPLD2 patients $(4.6 \mathrm{ng} / \mathrm{mL}$ and 6.9 ng/mL, respectively; $P=1.00$ ), but were higher in OND patients (median: $49.6 \mathrm{ng} / \mathrm{mL}, P<0.0001$ ). In contrast, irisin levels were similar in the OND and FPLD2 groups (median: $934 \mathrm{ng} / \mathrm{mL}$ and 944 ng/mL, respectively; $P=1.00$ ), but lower in the $\mathrm{HC}$ (median: $804 \mathrm{ng} / \mathrm{mL}, P<0.01$ vs OND and $P<$ 0.005 vs FPLD2). Interestingly, irisin/leptin ratios were similar in the HC and FPLD2 (median: 166 and 137, respectively; $P=1.00$ ), but strikingly lower in OND patients (median: $21, P<0.0001$; Table I, Fig. 1).

\section{Influence of gender}

No significant difference was observed in circulating irisin levels according to gender. In addition, a comparison of the three groups restricted to female-only patients served to reinforce certain group differences that had previously shown only borderline degrees of significance (Table S1; see supplementary materials associated with this article online). Interestingly, lean mass indices became significantly different between the HC and FPLD2 groups. Furthermore, BMI, intra-abdominal fat mass, intra-abdominal/total abdominal fat surface area ratio, FBG, triglycerides and CRP were the 
parameters that better differentiated the three female-only groups, with a trend favouring lean body mass. Nevertheless, gender did not modify the results for adipo-myokines leptin and irisin.

\section{Influence of diabetes status}

Comparison of the different parameters between diabetic $(n=11)$ and non-diabetic $(n=8)$ patients within the FPLD2 group showed significantly elevated glucose parameters in those with than without diabetes: FBG $(P<0.0001)$; insulin $(P=0.033)$; HbA1c $(P=0.001)$; and ALT $(P=0.028)$ (Table S2; see supplementary materials associated with this article online). Irisin/leptin ratio $(P=0.04)$, lean mass index $(P=0.039)$ and intra-abdominal fat levels $(P=0.027)$ were also significantly higher in the diabetic $v s$ non-diabetic FPLD2 patients. Furthermore, the diabetic group tended to be older $(P=$ 0.09). HDL $(P=0.047)$ and LDL $(P=0.039)$ cholesterol levels were slightly, but significantly, higher in the non-diabetic $v s$ diabetic group, whereas body weight, BMI, fat or lean mass in grammes or percentages, total abdominal fat and intra-abdominal/total abdominal fat surface area ratio, and irisin and leptin levels did not differ significantly between diabetic and non-diabetic FPLD2 patients (Table S2).

\section{Correlation of circulating irisin with anthropometric and metabolic parameters}

For the entire study group $(n=51)$, irisin levels were positively correlated with lean mass indices (lean mass $/$ height $^{2}$ ) and intra-abdominal/total abdominal fat mass, as well as with insulin, triglycerides and total cholesterol $(\mathrm{r}=+0.400-0.489 ; P<0.01)$ and, to a lesser extent, with BMI, lean body mass, FBG and HbA1c $(r=+0.281-0.335 ; P<0.05$; Table II, Fig. 2$)$. In addition, irisin levels tended to correlate with LDL cholesterol and ALT $(P=0.06-0.08)$, but not with body fat or abdominal fat mass, HDL cholesterol, AST, leptin and CRP.

\section{FPLD2 scores in the female-only groups}

Distribution of the FPLD2 score in patients after exclusion of male subjects, and as defined by the equation irisin $-(12.5 \times$ leptin $)$ according to patient group, is shown in Fig. 3 A. The ROC curve of this female-only FPLD2 score is displayed in Fig. 3 B. The AUC of the FPLD2 score was 74.4\%, 
and can be used with various thresholds: for instance, if the female FPLD2 score is defined as > 863, then sensitivity is $57.9 \%$ and specificity is $93.5 \%$, whereas at a lower threshold of 650 , sensitivity is then around $90.0 \%$, while specificity falls to about $30 \%$.

\section{DISCUSSION}

The diagnosis of monogenic lipodystrophy syndromes remains a difficult challenge. T2DM, of polygenic origin, may be associated with partial lipodystrophy, a limited capacity for fat storage in the limbs and insulin resistance [32], whereas monogenic forms of partial lipodystrophy syndromes with subtle clinical lipodystrophy have also been described [14-17]. Because muscle hypertrophy and/or altered metabolism are consistently associated with subcutaneous lipoatrophy in LMNA-related FPL2, our hypothesis was that biomarkers such as leptin, mainly secreted by adipose tissue, and irisin, recently identified as both an adipokine and a myokine, might help to distinguish patients with FPLD from patients with obesity.

Indeed, as expected, our three study groups (FPLD2, OND, HC), all of whom differed in terms of their qualitative or quantitative fat anomalies, showed significant differences in all anthropometric parameters. However, besides BMI, intra-abdominal fat mass was the only other parameter that clearly differentiated FPLD2 from either the HC or OND patients. Yet, when only female patients were assessed, the intra-abdominal/total abdominal fat ratio was also significantly different in the three groups: this ratio in the FPLD2 group was two- to threefold higher than in the HC and OND patients, thereby pointing to a specific subcutaneous lipoatrophy characterizing the disease. Although this was previously described in FPLD2 patients vs normal-weight HC [33], our study demonstrates for the first time that the intra-abdominal/total abdominal fat ratio also differs significantly between female FPLD2 and OND subjects, thereby confirming that this fat mass imbalance is a characteristic feature of FPLD.

On the other hand, lean mass percentages and indices differed or tended to differ between the HC and FPLD2 compared with the OND, with a higher lean body mass percentage and lower lean mass index in the HC and FPLD2 vs OND. In addition, when only female patients were considered, lean body mass then also differed significantly in our three groups, with values increasing from $\mathrm{HC}$ to 
FPLD2 patients and from FPLD2 to OND patients. These results confirm the increased skeletal muscle volume previously shown in FPLD2 women, albeit using a different technique, in comparison to $\mathrm{HC}$ [4]. Our study also shows that the lean body mass and indices of FPLD2 patients were intermediate to those of the $\mathrm{HC}$ and OND groups, and may be more discriminating than the intraabdominal/total abdominal fat ratio to differentiate female HC from female FPLD2 patients.

Interestingly, lean mass index and intra-abdominal fat surface area were the only anthropometric parameters to differ according to diabetes status among FPLD2 patients. In fact, the increased intra-abdominal fat surface area in diabetic FPLD2 patients may be related to the known relationship between visceral fat and insulin resistance. In addition, the higher lean mass indices and blood insulin levels in diabetic vs non-diabetic patients are in accordance with the significantly positive correlation between appendicular skeletal muscle indices and clinical parameters of insulin resistance (and also beta-cell function) previously demonstrated elsewhere [34-36]. This relationship is usually weaker in women, who have less muscle mass, and in ageing people because of age-related sarcopenia. In the present study, the relationship between lean body mass and insulin resistance could have been influenced by the more severe metabolic phenotype reported in female compared with male FPLD2 patients, and by the fact that our diabetic patients tended to be older $(P=0.09)$. Overall, our results suggest a close relationship between insulin resistance, visceral fat and muscle mass.

In addition, our study determined circulating levels of the adipokine leptin and adipo-myokine irisin in our three study groups (FPLD2, OND, HC), and found that leptin levels did not discriminate between HC and FPLD2 patients in accordance with their similar total fat mass, not even when only female patients were considered. Our results thereby confirm that leptin serum levels correlate with the amount of adipose tissue in patients with FPLD2 [18].

Both irisin levels and lean body mass and indices were, or tended to be, higher in OND and FPLD2 patients than in HC in our study, with a significant correlation between irisin and lean mass index as assessed by DXA. These results, which were independent of gender and diabetes status, are in accordance with the positive correlation previously observed between circulating irisin levels and skeletal muscle mass assessed by impedancemetry [37]. Finally, not only leptin levels, but also irisin/leptin ratios, can strikingly discriminate between FPLD2 and OND, but not between FPLD2 and 
HC. Irisin/leptin ratios were also higher in diabetic $v s$ non-diabetic FPLD2 patients, although irisin did not correlate with leptin in our study. In fact, conflicting results have been published regarding the relationship between irisin and leptin [38, 39]. However, a recent study of patients treated with metreleptin revealed that leptin in itself does not alter circulating irisin levels [40]. Importantly, our present results also show that irisin levels are not related to either total fat mass as assessed by body fat indices or total abdominal fat surface area, but are positively correlated with the intraabdominal/total abdominal fat ratio assessed by MRI. Nevertheless, whether irisin is secreted differently by visceral and by subcutaneous fat remains an open question. Irisin could be a marker of both lean body mass index and visceral distribution of fat mass, given its known role in subcutaneous adipocyte 'beiging/browning' [41-43]. It might also perhaps serve as an indicator of the optimal ratio between lean mass and visceral fat mass.

Given our present results, a biological diagnostic score was developed for female FPLD2 patients, determined by the equation irisin $-(12.5 \times$ leptin $)$, in an attempt to differentiate female patients with $L M N A$-associated FPLD2 from HC and OND patients. Validation of this female FPLD2 score $v s$ the gold standard (genetic testing) showed a good result (AUC: $74.5 \%$ ), and a threshold of 650 could be used as a screening test, given its sensitivity of $90 \%$ and specificity of $29 \%$. In our study sample, this score was able to distinguish between FPLD2 patients and normal-weight HC as well as patients with obesity. However, because of the small total number of patients, our study was set up and evaluated in the same sample. Nevertheless, this score should now be studied in a representative and larger cohort to confirm its relevance and to identify the most relevant threshold.

In the present study, circulating irisin levels were increased in FPLD2 patients, who were characterized by high lean body mass regardless of their fat mass. However, some limitations should be acknowledged when interpreting these results. First, there is as yet no consensus on the identification of the soluble portion of the irisin precursor FNDC5 membrane protein, as the mechanisms of secretion and the variable presence of target epitopes differ among manufacturers [20, 30]. Second, the size of each of our study groups was small and, finally, no conclusions regarding cause-effect relationships can be drawn. Clearly, further clinical studies to investigate the molecular mechanisms regulating irisin secretion, site(s) of action and signalling pathways are now necessary. 
That said, our present results suggest that the cross-talk between skeletal muscle and adipose tissue mediated by irisin could be contributing to the muscle hypertrophy seen in FPLD2, along with other mechanisms such as insulin resistance, intramyocellular lipids, the effects of hyperinsulinaemia on insulin-like growth factor (IGF)-1 receptors, and intrinsic defects of skeletal muscle metabolism due to lamin $\mathrm{A}$ and $\mathrm{C}$ dysfunction.

In conclusion, in addition to an increased intra-abdominal/total abdominal fat ratio $>0.5$, which differentiates women with FPLD2 from those with OND and HC, FPLD2 might also be differentiated from obese subjects at the biological level by lower leptin and higher irisin/leptin levels. FPLD2 can also be discriminated from healthy individuals by higher lean body mass indices and irisin levels, although the latter levels are similar to those of obese subjects. The FPLD2 score, derived by the equation irisin $-(12.5 \times$ leptin $)$, may also be proposed as a marker for female FPLD2 patients with a specificity $>90 \%$ when using a threshold $>863$. 
Disclosure statement: The authors have nothing to disclose

Clinical Trial Registration Number: Clin.gov2009-AO-1169-48

\section{ACKNOWLEDGEMENTS}

This study was supported by the French Ministry of Health (PHRC 2009; Clinical Trial Registration Number: Clin.gov2009-AO-1169-48). In addition, the authors are indebted to Hélène Verstavel and Anne-Sophie Vanceulebroek (clinical research nurses), Mr Patrick Gelé of the Center of Research Biology (CRB), the staff of the Department of Endocrinology, Diabetology and Metabolism, Department of Clinical Research and Délégation à la Recherche Clinique of Lille University Hospital. We also thank the endocrinologists of the Diamenord-AEDNL regional network. Our team is part of the European Consortium of Lipodystrophies (ECLIP) and French Rare Diseases Healthcare Network (FIRENDO) Rare Pathologies of Insulino-Resistance and Insulin Secretion (PRISIS).

\section{Appendix supplementary material}

Supplementary materials (Tables S1 and S2) associated with this article can be found at http://www.scincedirect.com at doi ... 


\section{REFERENCES}

1. Brown RJ, Araujo-Vilar D, Cheung PT, Dunger D, Garg A, Jack M, et al. The Diagnosis and Management of Lipodystrophy Syndromes: A Multi-Society Practice Guideline. J Clin Endocrinol Metab 2016; 101:4500-11.

2. Garg A. Lipodystrophies: genetic and acquired body fat disorders. J Clin Endocrinol Metab 2011; 96:3313-25.

3. Vantyghem MC, Balavoine AS, Douillard C, Defrance F, Dieudonne L, Mouton F, et al. How to diagnose a lipodystrophy syndrome. Ann Endocrinol (Paris) 2012; 73:170-89.

4. Ji H, Weatherall P, Adams-Huet B, Garg A. Increased skeletal muscle volume in women with familial partial lipodystrophy, Dunnigan variety. J Clin Endocrinol Metab 2013; 98:E1410-3.

5. Spuler S, Kalbhenn T, Zabojszcza J, van Landeghem FKH, Ludtke A, Wenzel K, et al. Muscle and nerve pathology in Dunnigan familial partial lipodystrophy. Neurology 2007; 68:677 $\square 83$.

6. Vantyghem MC, Pigny P, Maurage CA, Rouaix-Emery N, Stojkovic T, Cuisset JM, et al. Patients with familial partial lipodystrophy of the Dunnigan type due to a LMNA R482W mutation show muscular and cardiac abnormalities. J Clin Endocrinol Metab 2004; 89:533746.

7. Boguslavsky RL, Stewart CL, Worman HJ. Nuclear lamin A inhibits adipocyte differentiation: implications for Dunnigan-type familial partial lipodystrophy. Hum Mol Genet 2006; 15:653ロ63.

8. Perovanovic J, Dell'Orso S, Gnochi VF, Jaiswal JK, Sartorelli V, Vigouroux C, et al. Laminopathies disrupt epigenomic developmental programs and cell fate. Sci Transl Med 2016; 20; 8:335ra58

9. Vadrot N, Duband-Goulet I, Cabet E, Attanda W, Barateau A, Vicart P, et al. The p.R482W substitution in A-type lamins deregulates SREBP1 activity in Dunnigan-type familial partial lipodystrophy. Hum Mol Genet 2015; 24:2096-109. 
10. Le Dour C, Wu W, Béréziat V, Capeau J, Vigouroux C, Worman HJ. Extracellular Matrix Remodeling and Transforming Growth Factor- $\beta$ Signaling Abnormalities Induced by Lamin A/C Variants that Cause Lipodystrophy. J Lipid Res 2016 Nov 14. pii: jlr.M071381.

11. Béréziat V, Cervera P, Le Dour C, Verpont MC, Dumont S, Vantyghem MC, et al. LMNA mutations induce a non-inflammatory fibrosis and a brown fat-like dystrophy of enlarged cervical adipose tissue. Am J Pathol 2011;179: 2443-53.

12. Seale P, Bjork B, Yang W, Kajimura S, Chin S, Kuang S, et al. PRDM16 controls a brown fat/skeletal muscle switch. Nature 2008; 454:961ロ7.

13. Billon N, Dani C. Developmental origins of the adipocyte lineage: new insights from genetics and genomics studies. Stem Cell Rev 2012; 8:55-66.

14. Decaudain A, Vantyghem M-C, Guerci B, Hécart A-C, Auclair M, Reznik Y, et al. New metabolic phenotypes in laminopathies: LMNA mutations in patients with severe metabolic

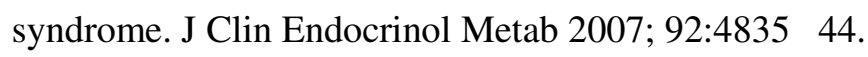

15. Visser ME, Kropman E, Kranendonk ME, Koppen A, Hamers N, Stroes ES, et al. Characterisation of non-obese diabetic patients with marked insulin resistance identifies a novel familial partial lipodystrophy-associated PPAR $\gamma$ mutation (Y151C). Diabetologia. $2011 ; 54: 1639 \square 44$.

16. Strickland LR, Guo F, Lok K, Garvey WT. Type 2 diabetes with partial lipodystrophy of the limbs: a new lipodystrophy phenotype. Diabetes Care.2013; 36: 2247-53.

17. Dutour A, Roll P, Gaborit B, Courrier S, Alessi MC, Tregouet DA, et al. High prevalence of laminopathies among patients with metabolic syndrome. Hum Mol Genet 2011; 20:3779-86.

18. Haque WA, Shimomura I, Matsuzawa Y, Garg A. Serum adiponectin and leptin levels in patients with lipodystrophies. J Clin Endocrinol Metab 2002; 87:2395.

19. Boström P, Wu J, Jedrychowski MP, Korde A, Ye L, Lo JC, et al. A PGC1- $\alpha$-dependent myokine that drives brown-fat-like development of white fat and thermogenesis. Nature 2012; $481: 463 \square 8$. 
20. Jedrychowski MP, Wrann CD, Paulo JA, Gerber KK, Szpyt J, Robinson MM, et al. Detection and Quantitation of Circulating Human Irisin by Tandem Mass Spectrometry. Cell Metab $2015 ; 22: 734-40$.

21. Shan T, Liang X, Bi P, Kuang S. Myostatin knockout drives browning of white adipose tissue through activating the AMPK-PGC1 $\alpha$-Fndc5 pathway in muscle. FASEB J 2013; 27:1981 $\square$ 9.

22. Huh JY, Dincer F' Mesfum E, Mantzoros CS. Irisin stimulates muscle growth-related genes and regulates adipocyte differentiation and metabolism in humans. Int $\mathrm{J}$ Obes (Lond) 2014;38:1538-44.

23. Moreno-Navarrete JM, Ortega F, Serrano M, Guerra E, Pardo G, Tinahones F, et al. Irisin is expressed and produced by human muscle and adipose tissue in association with obesity and insulin resistance. J Clin Endocrinol Metab 2013; 98:E769 $\square 78$.

24. Roca-Rivada A, Castelao C, Senin LL, Landrove MO, Baltar J, Belén Crujeiras A, et al. FNDC5/irisin is not only a myokine but also an adipokine. PloS One. 2013; 8:e60563.

25. Yan B, Shi X, Zhang H, Pan L, Ma Z, Liu S, et al. Association of serum irisin with metabolic syndrome in obese Chinese adults. PloS One 2014; 9:e94235.

26. Kurdiova T, Balaz M, Vician M, Maderova D, Vlcek M, Valkovic L, et al. Effects of obesity, diabetes and exercise on Fndc5 gene expression and irisin release in human skeletal muscle and adipose tissue: in vivo and in vitro studies. J Physiol 2014; 592:1091-107.

27. Stengel A, Hofmann T, Goebel-Stengel M, Elbelt U, Kobelt P, Klapp BF. Circulating levels of irisin in patients with anorexia nervosa and different stages of obesity--correlation with body

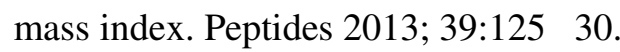

28. Crujeiras AB, Pardo M, Arturo R-R, Navas-Carretero S, Zulet MA, Martínez JA, et al. Longitudinal variation of circulating irisin after an energy restriction-induced weight loss and following weight regain in obese men and women. Am J Hum Biol 2014; 26:198ø207.

29. Højlund K, Boström P. Irisin in obesity and type 2 diabetes. J Diabetes Complications 2013; $27: 303 \square 4$.

30. Albrecht E, Norheim F, Thiede B, Holen T, Ohashi T, Schering L, et al. Irisin - a myth rather 
than an exercise-inducible myokine. Sci Rep 2015; 5:8889.

31. Carboni N, Politano L, Floris M, Mateddu A, Solla E, Olla S, et al. Overlapping syndromes in laminopathies: a meta-analysis of the reported literature. Acta Myologica 2013; 32: 7-17.

32. Lotta LA, Gulati P, Day FR, Payne F, Ongen H, van de Bunt M, et al. . Integrative genomic analysis implicates limited peripheral adipose storage capacity in the pathogenesis of human insulin resistance. Nat Genet 2017; 49:17-26.

33. Al-Attar SA, Pollex RL, Robinson JF, Miskie BA, Walcarius R, Little CH, et al. Quantitative and qualitative differences in subcutaneous adipose tissue stores across lipodystrophy types shown by magnetic resonance imaging. BMC Med Imaging 2007;12 : 7-13.

34. Sakai S, Tanimoto K, Imbe A, Inaba Y, Shishikura K, Tanimoto Y, et al. Decreased $\beta$-Cell Function Is Associated with Reduced Skeletal Muscle Mass in Japanese Subjects without Diabetes. PLoS One 2016; 11:e0162603.

35. Shishikura K, Tanimoto K, Sakai S, Tanimoto Y, Terasaki J, Terasaki J, et al. Association between skeletal muscle mass and insulin secretion in patients with type 2 diabetes mellitus. Endocr J 2014; 61: 281-7.

36. Moreno-Navarrete JM, Ortega F, Serrano M, Guerra E, Pardo G, Tinahones F, et al. Irisin is expressed and produced by human muscle and adipose tissue in association with obesity and insulin resistance. J Clin Endocrinol Metab 2013; 98: E769-78.

37. Hwang YC, Jeon WS, Park CY, Youn BS. The ratio of skeletal muscle mass to visceral fat area is a main determinant linking circulating irisin to metabolic phenotype. Cardiovasc Diabetol 2016; 15:9.

38. Palacios-González B, Vadillo-Ortega F, Polo-Oteyza E, Sánchez T, Ancira-Moreno M, Romero-Hidalgo S, et al. Irisin levels before and after physical activity among school-age children with different BMI: a direct relation with leptin. Obesity (Silver Spring) 2015; 23:729-32.

39. Blüher S, Panagiotou G, Petroff D, Markert J, Wagner A, Klemm T, et al. Effects of a 1-year exercise and lifestyle intervention on irisin, adipokines, and inflammatory markers in obese children. Obesity (Silver Spring) 2014;22:1701-8. 
40. Gavrieli A, Panagiotou G, Mantzoros CS. Leptin administration in physiological or pharmacological doses does not alter circulating irisin levels in humans. Int $\mathbf{J}$ Obes (Lond) 2016; 40:1461-3.

41. Vaughan RA, Gannon NP, Mermier CM, Conn CA. Irisin, a unique non-inflammatory myokine in stimulating skeletal muscle metabolism. J Physiol Biochem 2015; 71:679-89.

42. Zhang Y, Xie C, Wang H, Foss RM, Clare M, George EV, et al. Irisin exerts dual effects on browning and adipogenesis of human white adipocytes. Am J Physiol Endocrinol Metab 2016; 311:E530-41.

43. Rodríguez A, Becerril S, Ezquerro S, Méndez-Giménez L, Frühbeck G. Crosstalk between adipokinesnd myokines in fat browning. Acta Physiol (Oxf) 2017; 219:362-81. 


\section{Figure legends}

Fig. 1. Comparison of three groups of subjects (HC: healthy controls, OND: obese nondiabetic; FPLD2: LMNA-associated lipodystrophy) according to median levels of (A) body mass index (BMI), (B) fat mass index, (C) leptin, (D) intra-abdominal/total abdominal fat mass, (E) lean mass index, and (F) irisin. Levels of significance refer to all three groups.

Fig. 2. Correlation between blood irisin levels and lean mass index in the entire study population. HC: healthy controls; OND: obese non-diabetic; FPLD2: LMNA-associated lipodystrophy.

Fig. 3. (A) LMNA-associated lipodystrophy (FPLD2) score distribution; and (B) FPLD2 score receiver operating characteristic (ROC) curve. OND: obese non-diabetic; HC: healthy controls. 


\section{Fig $1 \mathrm{BMI}\left(\mathrm{kg} / \mathrm{m}^{2}\right)$}

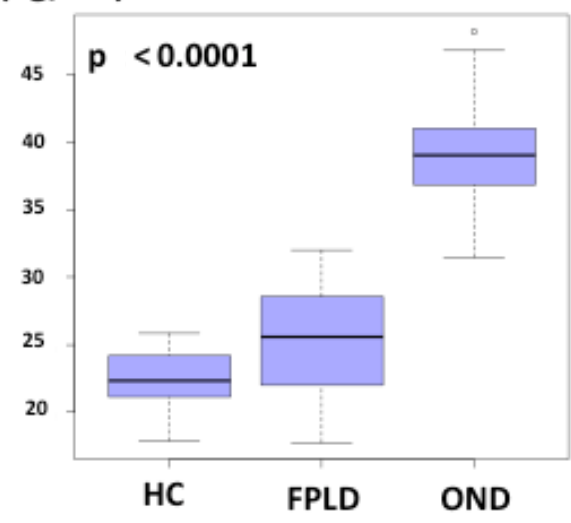

Fat mass $/$ height $^{2}\left(\mathrm{~g} / \mathrm{m}^{2}\right)$

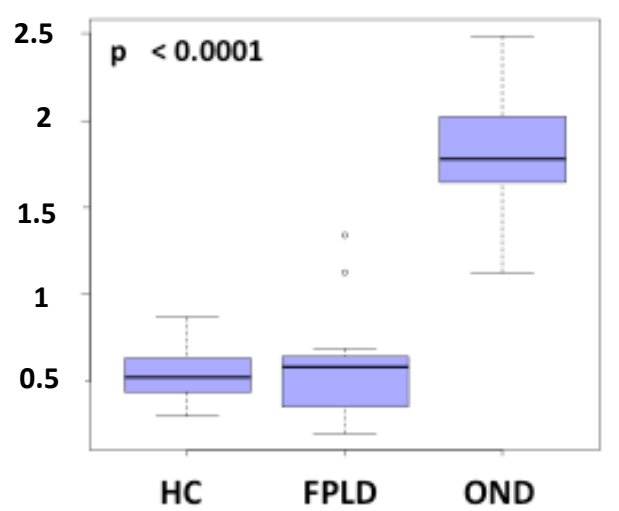

Leptin $(\mathrm{ng} / \mathrm{mL})$

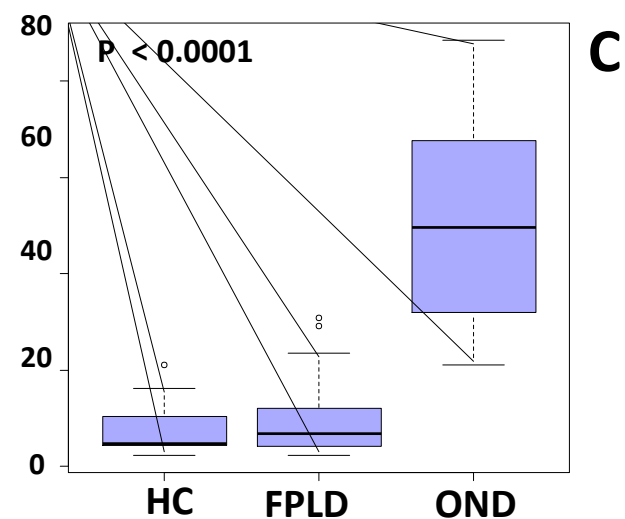

B

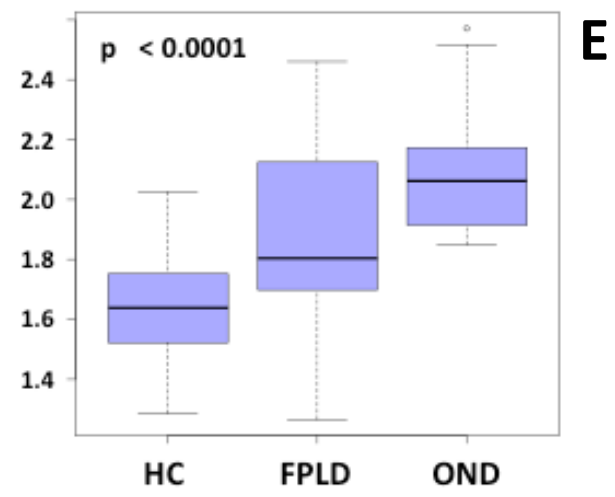

Irisin (ng/mL)

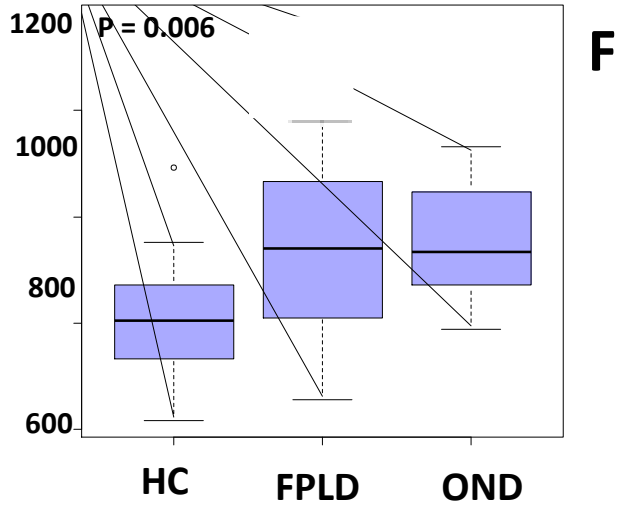




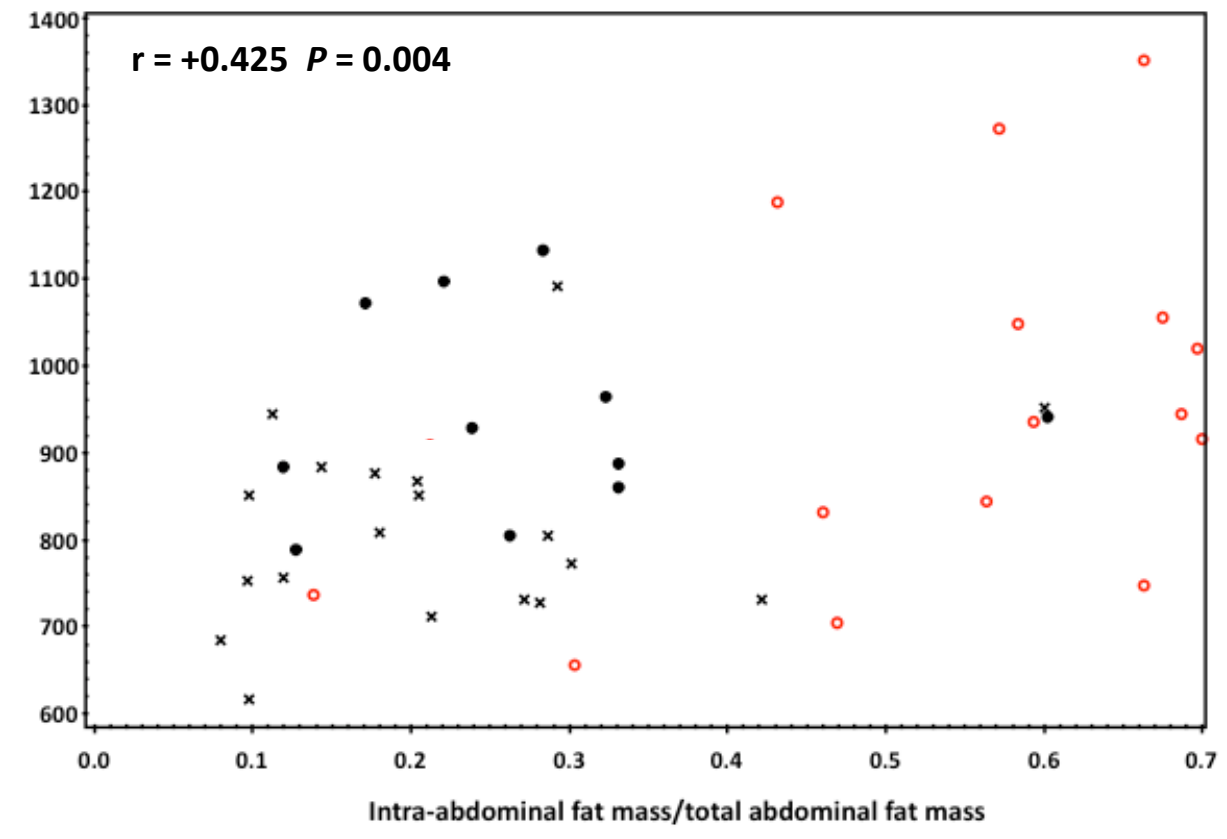

A

$\operatorname{lrisin}(\mathrm{ng} / \mathrm{mL})$

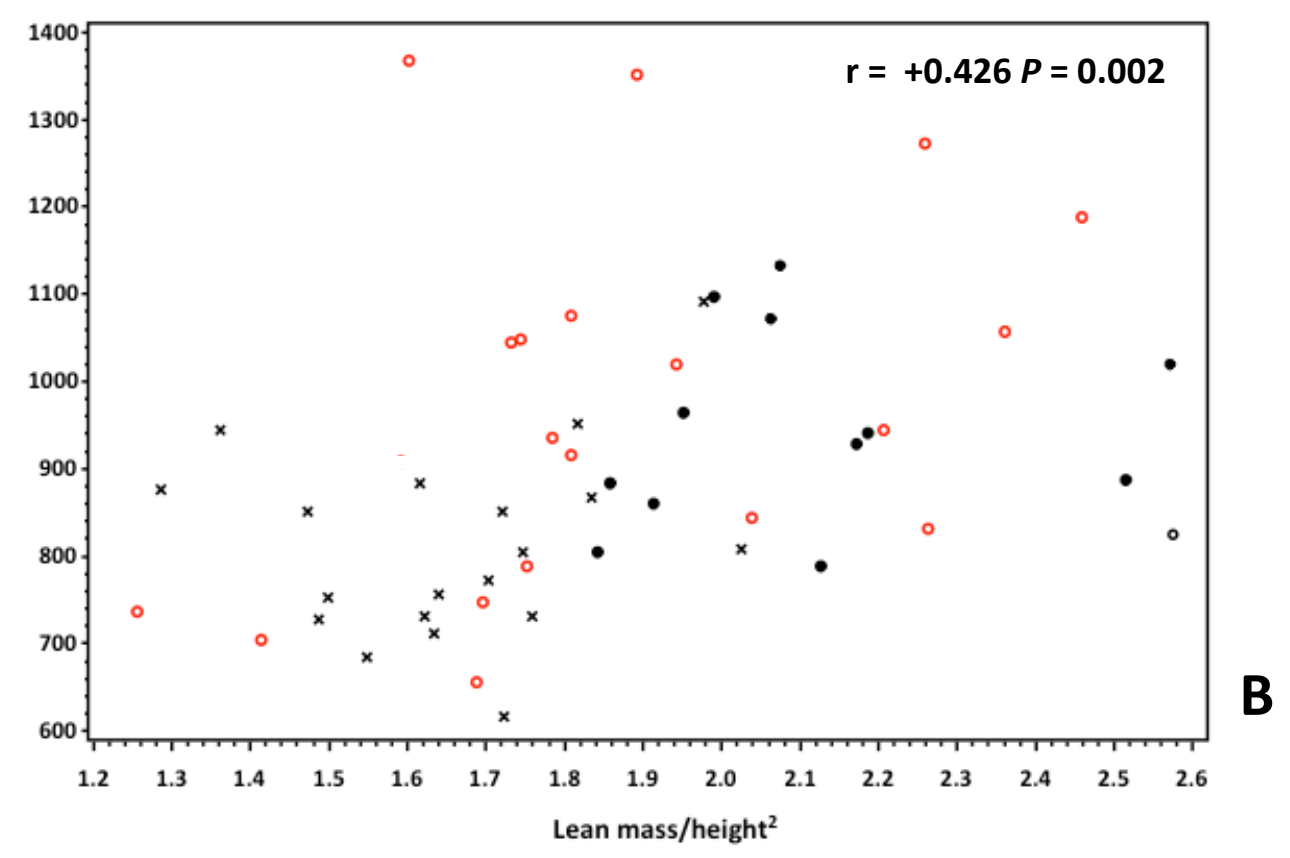


Score FPLD2 : irisin - (12.5 x leptin)

Sensitivity

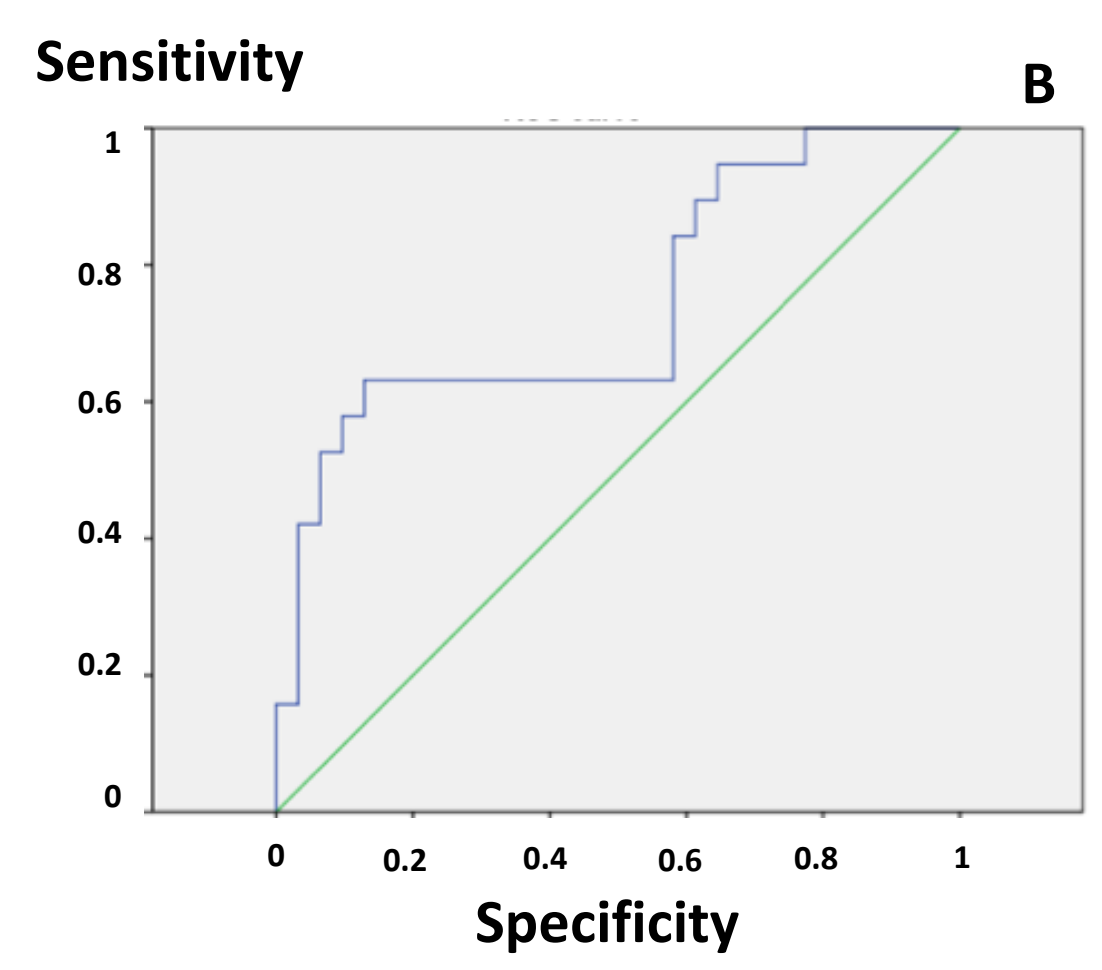

\section{Score FPLD2}

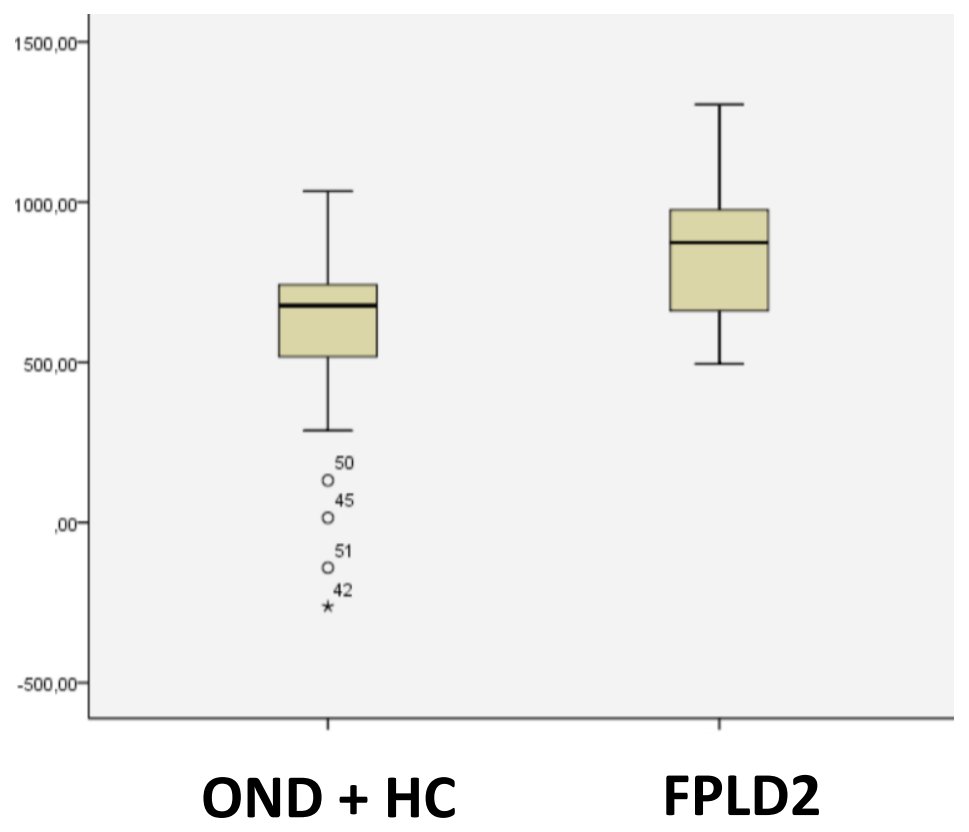

A

OND + HC

FPLD2
Fig 3 
Table I

Anthropometric, metabolic and inflammatory parameters in healthy controls (HC), and obese non-diabetic (OND) and familial partial lipodystrophy (FPLD2) patients expressed as medians [first and third quartiles]: parameters shown in italics can better differentiate the three group

\begin{tabular}{|c|c|c|c|c|c|c|c|}
\hline Parameters & $\mathrm{HC}$ & OND & FPLD2 & $P($ all $)$ & $P($ HC/OND $)$ & $P($ HC/FPLD2) & $P($ OND/FPLD2 $)$ \\
\hline Total, n & 19 & 13 & 19 & & & & \\
\hline \multicolumn{8}{|l|}{ ANTHROPOMETRIC } \\
\hline Age & $37[23 ; 55]$ & $51[45 ; 56]$ & $45[33 ; 58.5]$ & 0.22 & - & - & - \\
\hline Male gender, $\mathrm{n}$ & 10 & 2 & 3 & 0.04 & & & \\
\hline Body mass index $\left(\mathrm{kg} / \mathrm{m}^{2}\right)$ & $22[21 ; 24]$ & $39[37 ; 41]$ & $26[23 ; 29]$ & $<0.0001$ & $<0.0001$ & 0.02 & $<0.0001$ \\
\hline \multicolumn{8}{|l|}{ Body composition (DXA) } \\
\hline Fat mass $(\mathrm{g})$ & $15,620[11,898 ; 19,133]$ & $48,102[44,694 ; 50,984]$ & $15,188[8603 ; 17,525]$ & $<0.0001$ & $<0.0001$ & 0.98 & $<0.0001$ \\
\hline Total fat mass (\%) & $22[20 ; 30]$ & $45[43 ; 49]$ & $21[16 ; 30]$ & $<0.0001$ & $<0.0001$ & 0.99 & $<0.0001$ \\
\hline Fat mass $/$ height $^{2}$ or index $\left(\mathrm{g} / \mathrm{m}^{2}\right)$ & $0.52[0.43 ; 0.63]$ & $1.78[1.64 ; 2.02]$ & $0.55[0.37 ; 0.65]$ & $<0.0001$ & $<0.0001$ & 1.000 & $<0.0001$ \\
\hline Lean mass $(\mathrm{g})$ & $46,691[42,531 ; 55,645]$ & $54,019[48,517 ; 58,248]$ & $47,626[41,923 ; 59,717)$ & 0.008 & $<0.0001$ & 0.72 & 0.16 \\
\hline Lean mass $(\%)$ & $73[66 ; 76]$ & $52[48 ; 54]$ & $76[65 ; 79]$ & $<0.0001$ & $<0.0001$ & 0.87 & $<0.0001$ \\
\hline Lean mass/height ${ }^{2}$ or index $\left(\mathrm{g} / \mathrm{m}^{2}\right)$ & $1.64[1.50 ; 1.80]$ & $2.06[1.91 ; 2.17]$ & $1.80[1.70 ; 2.2]$ & $<0.0001$ & $<0.0001$ & 0.12 & 0.08 \\
\hline \multicolumn{8}{|l|}{ Abdominal fat mass (MRI) } \\
\hline Total abdominal fat area $\left(\mathrm{cm}^{2}\right)$ & $276[166 ; 374]$ & $833[711 ; 937]$ & $233[160 ; 336]$ & $<0.0001$ & $<0.0001$ & 0.66 & $<0.0001$ \\
\hline Intra-abdominal fat area $\left(\mathrm{cm}^{2}\right)$ & $50[21 ; 92]$ & $182[122 ; 336]$ & $135[75 ; 169]$ & 0.0002 & 0.0006 & 0.017 & 0.02 \\
\hline Intra-abdominal/total abdominal fat area & $0.20[0.11 ; 0.30]$ & $0.25[0.15 ; 0.33]$ & $0.58[0.46 ; 0.67]$ & $<0.0001$ & 0.74 & $<0.0001$ & $<0.0001$ \\
\hline $\begin{array}{l}\text { Lean mass/height } / \text { intra-abdominal fat } \\
\text { area }\left(\mathrm{g} / \mathrm{m}^{2}\right) /\left(\mathrm{cm}^{2}\right)\end{array}$ & $0.03[0.02 ; 0.07]$ & $0.01[0.01 ; 0.02]$ & $0.01[0.01 ; 0.02]$ & 0.0005 & 0.0015 & 0.03 & 0.5 \\
\hline $\begin{array}{l}\text { Lean mass/height } / \text { total abdominal fat area } \\
\left(\mathrm{g} / \mathrm{m}^{2}\right) /\left(\mathrm{cm}^{2}\right)\end{array}$ & $0.006[0.004 ; 0.010]$ & $0.002[0.002 ; 0.003]$ & $0.007[0.006 ; 0.010]$ & $<0.0001$ & $<0.0001$ & 0.27 & $<0.0001$ \\
\hline \multicolumn{8}{|l|}{ METABOLIC } \\
\hline \multicolumn{8}{|l|}{ Glucose } \\
\hline Fasting blood glucose $(\mathrm{mmol} / \mathrm{L})$ & $4.73[4.61 ; 5.22]$ & $5.11[4.61 ; 5.50]$ & $6[4.78 ; 8.25]$ & 0.0005 & 0.87 & 0.0007 & 0.02 \\
\hline Fasting blood insulin $(\mathrm{pmol} / \mathrm{L})$ & $31[21 ; 40]$ & $50[31 ; 73]$ & $64[42 ; 110]$ & 0.01 & 0.07 & 0.009 & 0.18 \\
\hline HbAlc (\%) & $5[4.9 ; 5.6]$ & $5.8[5.4 ; 6.2]$ & $6.2[5.3 ; 7.1]$ & $<0.0001$ & 0.0001 & $<0.0001$ & 0.08 \\
\hline \multicolumn{8}{|l|}{ Liver transaminases (IU/L) } \\
\hline Aspartate aminotransferase & $22[19 ; 25]$ & $20[19 ; 25]$ & $29[22 ; 39]$ & 0.01 & 1.000 & 0.01 & 0.04 \\
\hline Alanine aminotransferase & $17[14 ; 25]$ & $20[19 ; 27]$ & $29[22 ; 50]$ & 0.005 & 0.089 & 0.003 & 0.13 \\
\hline \multicolumn{8}{|l|}{ Lipids (mmol/L) } \\
\hline Total cholesterol & $5.05[4.66 ; 5.49]$ & $4.86[4.53 ; 5.51]$ & $4.42[3.95 ; 5.40]$ & 0.85 & - & - & - \\
\hline Low-density lipoprotein cholesterol & $3.00[2.56 ; 3.36]$ & $3.10[2.64 ; 3.72]$ & $2.47[1.85 ; 3.47]$ & 0.55 & - & - & - \\
\hline High-density lipoprotein cholesterol & $1.45[1.16 ; 1.81]$ & $1.19[1.01 ; 1.47]$ & $1.05[0.74 ; 1.20]$ & 0.01 & 0.48 & 0.015 & 0.35 \\
\hline Triglycerides & $1.0[0.7 ; 1.3]$ & $1.5[1.2 ; 1.8]$ & $2.0[1.0 ; 2.6]$ & 0.0008 & 0.05 & 0.001 & 0.03 \\
\hline \multicolumn{8}{|l|}{ ADIPO-MYOKINES } \\
\hline Leptin $(\mathrm{ng} / \mathrm{mL})$ & $4.6[4.1 ; 10.7]$ & $49.6[31.9 ; 67.6]$ & $6.9[4.3 ; 13.5]$ & $<0.0001$ & $<0.0001$ & 0.20 & $<0.0001$ \\
\hline Irisin $(\mathrm{ng} / \mathrm{mL})$ & $804[732 ; 876]$ & $934[872 ; 1046]$ & $944[788 ; 1076]$ & 0.006 & 0.0099 & 0.005 & 0.68 \\
\hline Irisin/leptin ratio & $166[71 ; 214]$ & $21[13 ; 32]$ & $137[68 ; 183]$ & $<0.0001$ & $<0.0001$ & 0.45 & $<0.0001$ \\
\hline \multicolumn{8}{|l|}{ INFLAMMATORY } \\
\hline C-reactive protein $(\mathrm{mg} / \mathrm{L})$ & 0 & $7[3 ; 15]$ & $3[0.0 ; 3.5]$ & $<0.0001$ & $<0.0001$ & 0.0009 & 0.11 \\
\hline
\end{tabular}


Table II Correlation between blood irisin levels and anthropometric, metabolic and inflammatory parameters in the entire study group (healthy controls + obese non-diabetic + familial partial lipodystrophy subjects)

\begin{tabular}{|c|c|c|c|}
\hline Parameters & $\mathbf{n}$ & $\mathbf{r}$ & $\boldsymbol{P}$ \\
\hline \multicolumn{4}{|l|}{ ANTHROPOMETRIC } \\
\hline Body mass index $\left(\mathrm{kg} / \mathrm{m}^{2}\right)$ & 50 & +0.281 & 0.048 \\
\hline Fat mass (g), dual-energy X-ray absorptiometry & 45 & +0197 & 0.195 \\
\hline \multicolumn{4}{|l|}{$(\mathrm{DXA})$} \\
\hline Fat mass $(\%)$, DXA & 45 & +0.073 & 0.612 \\
\hline Lean mass $(\mathrm{g}), \mathrm{DXA}$ & 50 & $+\mathbf{0 . 3 3 5}$ & 0.017 \\
\hline Lean mass $(\%)$, DXA & 50 & -0.047 & 0.748 \\
\hline Lean mass $/$ height ${ }^{2}\left(\mathrm{~g} / \mathrm{m}^{2}\right)$, DXA & 50 & +0.426 & 0.002 \\
\hline Total abdominal fat area $\left(\mathrm{cm}^{2}\right)$, magnetic resonance & 45 & +0.078 & 0.610 \\
\hline \multicolumn{4}{|l|}{ imaging (MRI) } \\
\hline Intra-abdominal fat area $\left(\mathrm{cm}^{2}\right)$, MRI & 45 & +0.220 & 0.147 \\
\hline Intra-abdominal/total abdominal fat area, MRI & 45 & +0.425 & 0.004 \\
\hline \multicolumn{4}{|l|}{ METABOLIC } \\
\hline Fasting blood glucose & 50 & +0.304 & 0.032 \\
\hline Fasting blood insulin & 48 & +0.489 & $<0.0001$ \\
\hline $\mathrm{HbA} 1 \mathrm{c}$ & 50 & +0.315 & 0.026 \\
\hline Total cholesterol & 50 & +0.400 & 0.004 \\
\hline High-density lipoprotein cholesterol & 50 & -0.171 & 0.235 \\
\hline Low-density lipoprotein cholesterol & 50 & +0.249 & 0.082 \\
\hline Triglycerides & 50 & +0.442 & 0.001 \\
\hline Aspartate aminotransferase & 50 & +0.133 & 0.3 \\
\hline Alanine aminotransferase & 50 & +0.264 & 0.063 \\
\hline Leptin & 50 & +0.110 & 0.448 \\
\hline \multicolumn{4}{|l|}{ INFLAMMATORY } \\
\hline C-reactive protein & 50 & +0.009 & 0.953 \\
\hline
\end{tabular}

